\title{
Retrospective study of the ultrasound characteristics of the tibial nerve in patients with type 2 diabetic peripheral neuropathy
}

\author{
Kun Wang ${ }^{1,2 \#}$, Dong Yu ${ }^{3 \#}$, Taotao Yao ${ }^{4 \#}$, Shuchen Zhang ${ }^{5}$, Li Wen $^{6}$, Chenyao Gu ${ }^{5}$ \\ ${ }^{1}$ Department of Ultrasound, the Affiliated Hospital of Binzhou Medical University, Binzhou, China; ${ }^{2}$ Department of Ultrasound, Zhongshan \\ Hospital of Fudan University, Shanghai, China; ${ }^{3}$ Department of Ultrasound, North China Medical Treatment Health Group, Fengfeng General \\ Hospital, Handan, China; ${ }^{4}$ Rehabilitation Center, the First Affiliated Hospital of Guangzhou University of Chinese Medicine, Guangzhou, China; \\ ${ }^{5}$ Department of Ultrasound, Yancheng City, No. 1 People's Hospital, Yancheng, China; ${ }^{6}$ Department of Function, The Special Care Hospital of \\ Hebei Province, Shijiazhuang, China \\ Contributions: (I) Conception and design: D Yu, K Wang; (II) Administrative support: S Zhang; (III) Provision of study materials or patients: K Wang; \\ (IV) Collection and assembly of data: L Wen, C Gu; (V) Data analysis and interpretation: T Yao; (VI) Manuscript writing: All authors; (VII) Final \\ approval of manuscript: All authors. \\ "These authors contributed equally to this work. \\ Correspondence to: Dr. Shuchen Zhang. Department of Ultrasound, Yancheng City, No. 1 People's Hospital, 166 Yulong Road, Tinghu District, \\ Yancheng, China. Email: 2220099999@qq.com.
}

Background Ultrasound is a promising imaging examination for type 2 diabetic peripheral neuropathy (DPN). This study aimed to explore the risk factors of ultrasound diagnostic characteristics in the tibial nerves of patients with type 2 DPN.

Methods: The Michigan Neuropathy Screening Instrument (MNSI) was used for neuropathy evaluation, and 520 patients with type 2 diabetes were divided into a DPN group and non-DPN (NDPN) group with 2 points as the cut-off value. The two groups were matched at a ratio of 1:1 according to age, BMI, calf circumference, and gender, resulting in 44 matched pairs. The dependent variable was type 2 DPN and the concomitant variables were the width, thickness, cross-sectional area, and unclear honeycomb-like structure of the tibial nerve. A 1:1 matched conditional logistic regression model was established to analyze which ultrasound diagnostic characteristics of the tibial nerve were risk factors for type $2 \mathrm{DPN}$.

Results: The thickness $(\mathrm{OR}=5.176, \mathrm{P}=0.043)$ and cross-sectional area $(\mathrm{OR}=1.659, \mathrm{P}=0.030)$ of the tibial nerve were risk factors for the diagnosis of DPN, while the width and unclear honeycomb-like structure of the nerve were not $(\mathrm{P}>0.05)$. In the diagnosis of $\mathrm{DPN}$, the area under the receiver operating characteristic (ROC) curve of the cross-sectional area of the tibial nerve was 0.747 , and the sensitivity, specificity, positive predictive value, and negative predictive value were $61.36 \%, 95.45 \%, 93.10 \%$, and $71.20 \%$, respectively. The area under the ROC curve of tibial nerve thickness was 0.867 , and the sensitivity, specificity, positive predictive value, and negative predictive value were $81.82 \%, 90.91 \%, 90.00 \%$, and $83.30 \%$, respectively. The area under the ROC curve of thickness was larger than that of cross-sectional area of the tibial nerve ( $\mathrm{z}$ statistic $=1.800, \mathrm{P}=0.07)$.

Conclusions: The thickness and cross-sectional area of the tibial nerve measured by ultrasound are risk factors for type 2 DPN. The diagnostic sensitivity of the thickness is higher than the cross-sectional area, while the diagnostic specificity of the cross-sectional area is higher than the thickness.

Keywords: Diabetic peripheral neuropathy (DPN); ultrasound; tibial nerve; 1:1 matched conditional logistic regression model; receiver operating characteristic curve (ROC curve)

Submitted May 20, 2021. Accepted for publication Jul 28, 2021.

doi: 10.21037/apm-21-1573

View this article at: https://dx.doi.org/10.21037/apm-21-1573

(c) Annals of Palliative Medicine. All rights reserved. 


\section{Introduction}

The wide application of high-frequency linear array ultrasonic probe in recent years has made ultrasound more advantageous than other imaging examinations in the observation of superficial nerves (1), and its use in the diagnosis of diabetic peripheral neuropathy (DPN) holds great promise. In different subjects, the incidence of peripheral neuropathy was significantly different. It is so common for the diabetes peripheral neuropathy, which is up to $51 \%$ in type 2 diabetes patients. After the occurrence of peripheral neuropathy, the quality of life decreased and the economic burden increased in half patients with type 2 diabetes mellitus. Early stage intervention around type 2 diabetes is the key to diagnosis and treatment $(2,3)$. DPN is insidious, progresses slowly, and mainly manifests as sensory nerve involvement which may lead to severe sensory loss, painful and refractory ulcers, infections, and wounds that are difficult to heal and can eventually lead to amputation $(4,5)$. This study used 1:1 matched conditional logistic regression analysis to explore which tibial nerve ultrasound diagnostic characteristics were risk factors for DPN. We present the following article in accordance with the STARD reporting checklist (available at https://dx.doi.org/10.21037/ apm-21-1573).

\section{Methods}

\section{Participants}

The results of 452 patients with type 2 diabetes patients who underwent tibial nerve ultrasound examination from July 2018 to February 2021 were collected. The inclusion criteria were patients who, following the American Diabetes Association's latest diagnostic criteria for diabetes in 2017, showed glycosylated hemoglobin (HbA1c) $\geq 6.5 \%$, or fasting plasma glucose $(\mathrm{FPG}) \geq 7.0 \mathrm{mmol} / \mathrm{L}$, or oral glucose tolerance test (OGTT) $2 \mathrm{~h}$ blood glucose $\geq 11.1 \mathrm{mmol} / \mathrm{L} \mathrm{(6)}$.

The exclusion criteria were any of the following: type 1 diabetes, Guillain-Barré syndrome, severe cervical and lumbar neuropathy, rheumatoid arthritis, alcoholism, syphilis, tarsal duct tibial nerve entrapment syndrome, hereditary neuropathy, such as nerve fat infiltration, and a history of neurosurgery.

Neuropathy was evaluated using the Michigan Neuropathy Screening Instrument physical examination (MNSI-PE), and patients were divided into a DPN group and non-DPN (NDPN) group with 2 points as the cutoff value. Compared with other evaluation methods,
MNSI has the advantages of strong reliability, simple and quick operation, and good feasibility, so it is more suitable for early large-scale screening of DPN (6). Patients were matched in a 1:1 ratio according to age, BMI, calf circumference, and gender, resulting in 44 successfully matched pairs. The NDNP group was composed of 44 patients, including 27 males and 17 females, aged 39$73(54.772 \pm 7.278)$ years old, with a BMI of 19.24$26.27(22.880 \pm 1.624) \mathrm{kg} / \mathrm{m}^{2}$, calf circumference of $11.13-20.50(15.100 \pm 1.990) \mathrm{cm}$, and disease course of 2-6 (4.046 \pm 0.963$)$ years. In the DNP group there were 44 patients, including 27 males and 17 females, aged 44-73 (54.046 \pm 6.696$)$ years old, with a BMI of $19.91-26.89(22.996 \pm 1.373) \mathrm{kg} / \mathrm{m}^{2}$, calf circumference of 11.08-19.97 $(14.457 \pm 1.730) \mathrm{cm}$, and disease course of $2-16(8.841 \pm 8.779)$ years. The study was conducted in accordance with the Declaration of Helsinki (as revised in 2013). This study was reviewed and approved by the Ethics Committee of North China Medical Treatment Health Group Fengfeng General Hospital (20201-4-11), and all subjects participated in the study with informed consent.

\section{Apparatus and methods}

A Hitachi Aloka ARIETTA70 ultrasound diagnostic instrument was used in this study. A probe L44 was selected, the frequency was set to $5-18 \mathrm{MHz}$, the detection depth was $2 \mathrm{~cm}$, the mechanical index (MI) was 1.1-1.4, the dynamic contrast was 60-70, the tissue harmonic function was turned on, and the resolution was adjusted as priority. Subjects were placed in the supine position with the hips abducted, knees bent, heels slightly adducted, and toes externally rotated, and the thickness, width, and cross-sectional area of the tibial nerves were measured horizontally at the medial malleolus. Changes in tibial nerve echo were observed, and if the tibial nerve cross section had a honeycomb-like structure, but the nerve bundle structure could be displayed, 0 points were given, and if the nerve had a honeycomb-like structure and the display was blurred, 1 point was given.

To establish DPN evaluation criteria, the MNSI was used to perform physical examination on all subjects, with a full score of 10 . Feet were evaluated for deformity, dry skin, callus, infection, and ulceration with 0 points given for a normal foot appearance. Patients were given 1 point if any of these signs were present, and an additional 1 point if ulceration was present. Patients with a normal Achilles reflex were given 0 points, while a decreased Achilles reflex 


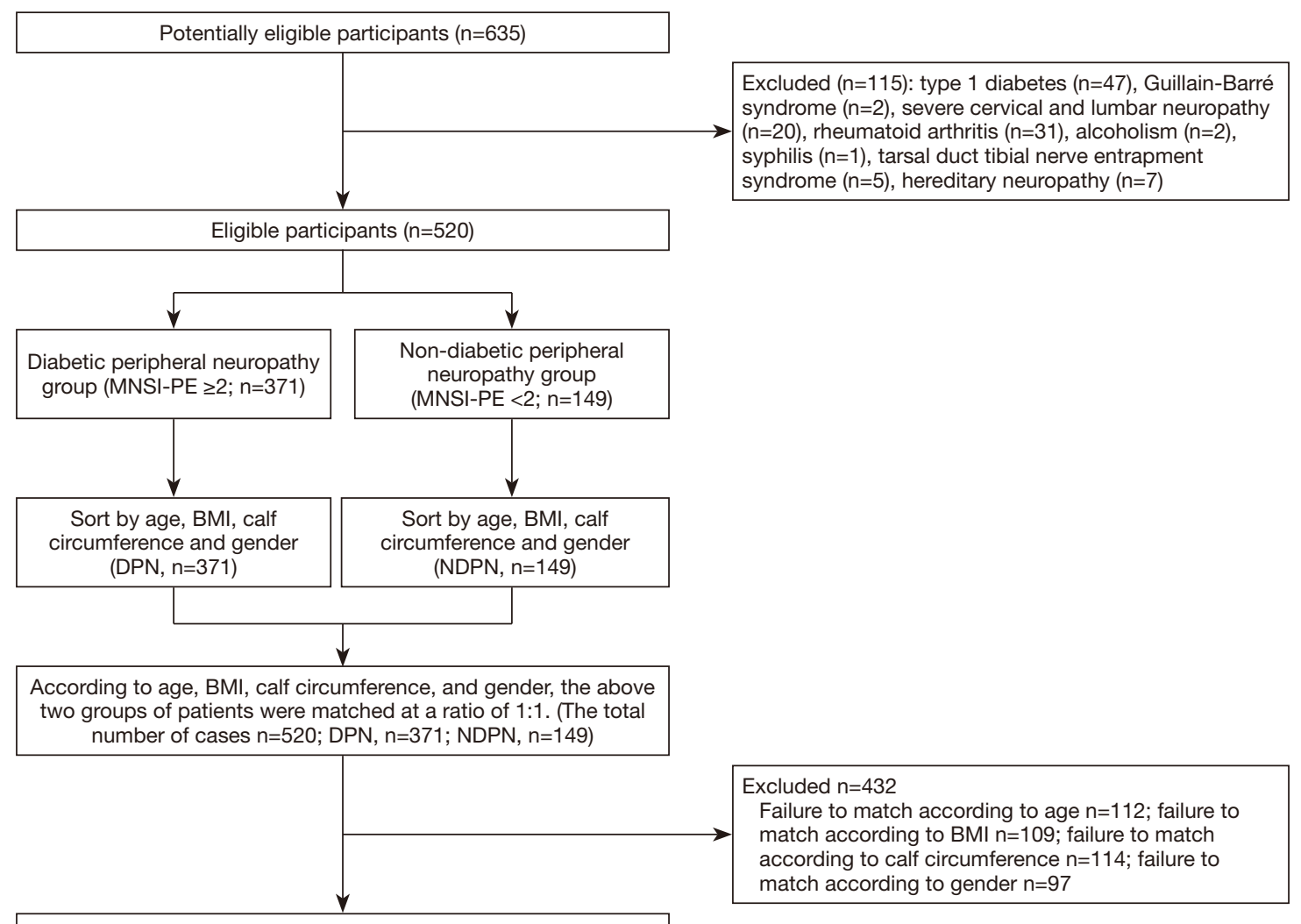

A total of 44 pairs were successfully matched $(n=88)$

Figure 1 Flow chart. MNSI-PE, Michigan Neuropathy Screening Instrument physical examination; DPN, diabetic peripheral neuropathy; NDPN, non-DPN.

attracted 0.5 points and an absent reflex 1 point. Patients with normal toe vibration sensation were given 0 points, those with a weakened vibration sensation were given 0.5 points, and those with no vibration sensation were given 1 point, and those with normal light touch were given 0 points, those with a mild moderate deficit were given 0.5 points, and those with severe deficit were given 1 point. Using 2 points as the diagnostic cut-off value, the patients were divided into a DPN group and NDPN group (Figure 1).

The measurement standard of the tibial nerve was established by selecting the nerve at the unilateral lower limb ankle canal as the measurement plane, and the probe placed perpendicular to the skin. The direction of the connection between the medial malleolus and the calcaneus was selected, and the closest distance between the tibial nerve and the medial malleolus point and calcaneus point was selected to measure the width of the tibial nerve. The distance perpendicular to the width measurement line of the thickest part of the tibial nerve was selected to measure its thickness, and the trajectory method was used to trace the epineurium which was identified by ultra-high-frequency (UHF) ultrasound to measure the cross-sectional area of the tibial nerve (Figure 2). The examination was performed by two experienced doctors who were trained in ultrasound examination of the tibial nerve.

\section{Statistical analysis}

SPSS 22.0 and MedCalc19.0 software were used in this study. Paired $t$-test and chi-square test were used to compare the general conditions of the two groups, and a 1:1 matched conditional logistic regression model was established. Receiver operating characteristic (ROC) curve analysis was used for model verification and $\mathrm{P}<0.05$ indicated differences were statistically significant. According to the number of observed variables, when the number of pairs was greater than 40, a logistics regression model was established. Cases 
with missing date were removed.

\section{Results}

\section{Comparison of general conditions}

There were no statistically significant differences between the two groups in gender, age, BMI, calf circumference, and tibial nerve width of the left and right legs $(\mathrm{P}>0.05)$. Compared with the NDPN group, the DPN group had a longer course of disease, larger tibial nerve thickness, and cross-sectional area $(\mathrm{P}<0.05$, two-sided) (Table 1, Figure 1).

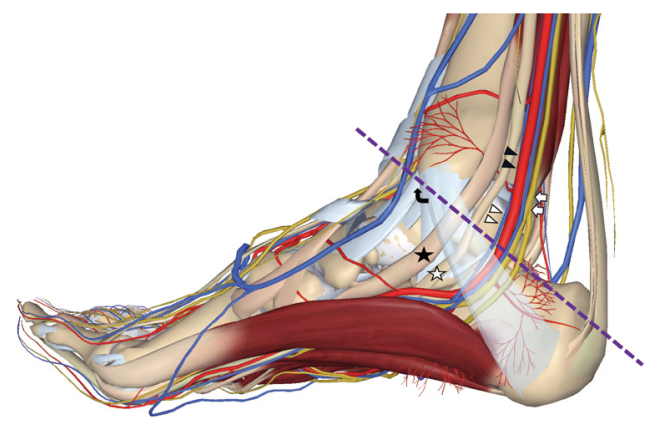

Figure 2 Anatomical diagram of the ankle: the line between the medial malleolus (bent arrow) and the calcaneus is a sectional view of ultrasound scan. Tibial nerve $(\uparrow)$, posterior tibial artery $(\triangle)$, posterior tibial vein $(\boldsymbol{\Delta})$ are deformed in the ankle canal. The

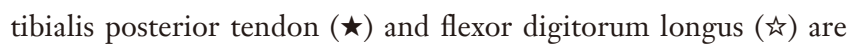
supplied by the tibial nerve L4-S3.

\section{Logistic regression analysis results and model verification}

This revealed tibial nerve thickness $(\mathrm{OR}=5.176, \mathrm{P}=0.043)$ and cross-sectional area $(\mathrm{OR}=1.659, \mathrm{P}=0.030)$ were risk factors for DPN, while the width of tibial nerve and an unclear honeycomb-like structure were not $(\mathrm{P}>0.05)$. The ROC was drawn using the predicted value of the ultrasound characteristic probability of DPN as the predicted value. The area under the ROC curve was 0.882 , the standard error was $0.054,95 \% \mathrm{CI}(0.805,0.999)$, and the sensitivity, specificity, positive predictive value, and negative predictive value were $81.82 \%, 95.45 \%, 94.70 \%$, and $84.00 \%$, respectively, indicating that the forecast was relatively accurate (Table 2). For the diagnosis of DPN, the area under the ROC curve of the cross-sectional area of the tibial nerve was 0.747 , and the sensitivity, specificity, positive predictive value, and negative predictive value are $61.36 \%, 95.45 \%$, $93.10 \%$, and $71.20 \%$, respectively. Further, the area under the ROC curve of tibial nerve thickness was 0.867 , and the sensitivity, specificity, positive predictive value, and negative predictive value were $81.82 \%, 90.91 \%, 90.00 \%$, and $83.30 \%$, respectively (Table 3, Figures 3-5).

\section{Discussion}

Ultrasound examination is an efficient examination, which is non-invasive, radiation-free, reliable and real-time. With the rapid development of ultrasonic transducer and the promotion of various efficient algorithms, the highresolution ultrasound equipment can clearly display the

Table 1 General data and ultrasonic examination results

\begin{tabular}{|c|c|c|c|c|}
\hline Group & NDPN & DPN & $\mathrm{F} / \chi^{2}$ & $\mathrm{P}$ \\
\hline Gender (male/female) & $27 / 17$ & $27 / 17$ & & \\
\hline Age (years) & $54.772 \pm 7.278$ & $54.046 \pm 6.696$ & -1.524 & 0.131 \\
\hline BMI $\left(\mathrm{kg} / \mathrm{cm}^{2}\right)$ & $22.880 \pm 1.624$ & $22.996 \pm 1.373$ & -0.263 & 0.793 \\
\hline Course of disease (year) & $4.046 \pm 0.963$ & $8.841 \pm 8.779$ & -3.602 & 0.001 \\
\hline Width (mm) & $4.848 \pm 0.732$ & $5.124 \pm 0.662$ & -1.858 & 0.067 \\
\hline Thickness (mm) & $3.525 \pm 0.098$ & $4.341 \pm 1.207$ & -4.471 & $<0.001$ \\
\hline Cross-sectional area $\left(\mathrm{mm}^{2}\right)$ & $20.087 \pm 0.999$ & $21.648 \pm 2.330$ & -4.085 & $<0.001$ \\
\hline
\end{tabular}

DPN, diabetic peripheral neuropathy; NDPN, non-DPN. 
Table 2 Conditional logistic regression analysis of the influencing factors of DPN

\begin{tabular}{lcccccc}
\hline Variables & B & Std. error & Wald & Sig & Exp(B) & 95\% Cl \\
\hline Width & 0.251 & 2.480 & 0.010 & 0.919 & 1.285 & $0.010-165.900$ \\
Thickness & 1.644 & 0.812 & 4.102 & 0.043 & 5.176 & $1.054-25.408$ \\
Cross-sectional area & 0.506 & 0.234 & 4.696 & 0.030 & 1.659 & $1.050-2.622$ \\
Honeycomb structure & -0.619 & 0.619 & 0.999 & 0.317 & 0.538 & $0.160-1.813$ \\
\hline
\end{tabular}

DPN, diabetic peripheral neuropathy.

Table 3 ROC curves of the observed variables in diabetic tibial neuropathy

\begin{tabular}{lcccccrr}
\hline Variables & AUC & SE & Sensitivity & Specificity & Positive predictive value & Negative predictive value & 95\% Cl \\
\hline Width & 0.518 & 0.0627 & 52.27 & 61.36 & 57.5 & 56.2 & $0.409-0.626$ \\
Thickness & 0.867 & 0.0467 & 81.82 & 90.91 & 90.00 & 83.30 & $0.778-0.930$ \\
Cross-sectional area & 0.747 & 0.0605 & 61.36 & 95.45 & 93.10 & 71.20 & $0.643-0.834$ \\
Honeycomb structure & 0.523 & 0.0524 & 40.91 & 63.64 & 52.90 & 51.90 & $0.414-0.630$ \\
\hline
\end{tabular}

ROC, receiver operating characteristic; AUC, area under the curve.

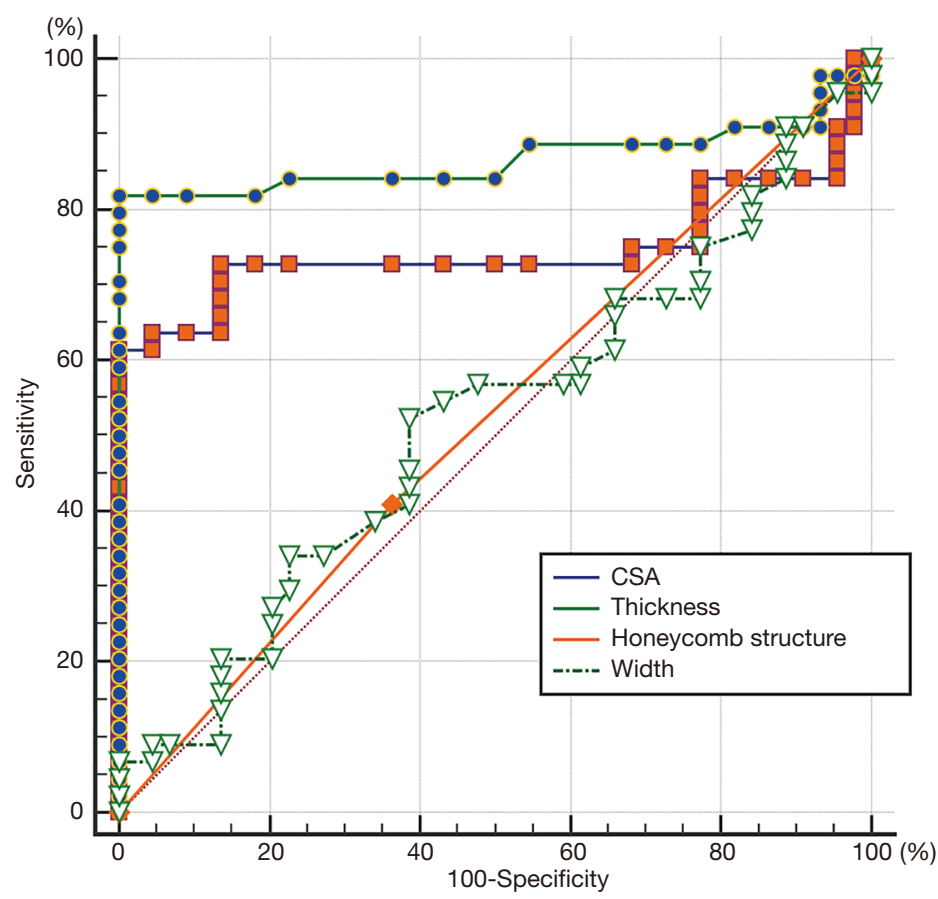

Figure 3 ROC curve of each observed variable. Ultrasonic characteristics include the following four variables: (I) CSA (the blue line); (II) thickness (the green line); (III) honeycomb structure (the orange line); (IV) width (the green dotted line). CSA, cross-sectional area.

subtle structure of the peripheral nerve without the need for contrast-enhanced ultrasound. Therefore, ultrasound examination has become the optimal imaging method of peripheral nerve examination.

While commonly used methods for the clinical diagnosis of DPN include ultrasound, natural electrophysiological 


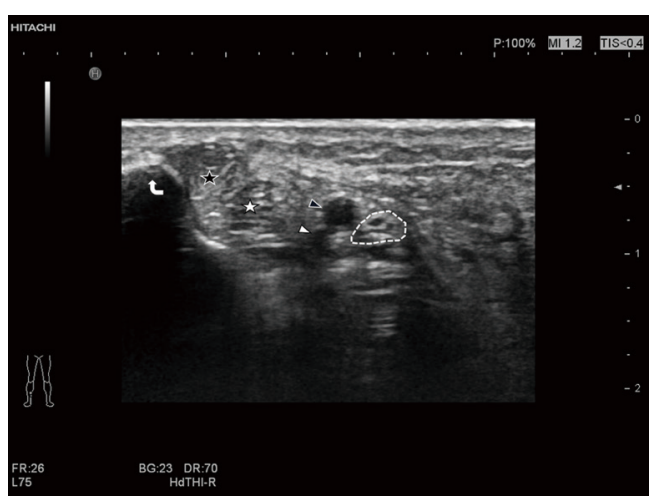

Figure 4 A 50-year-old man with type 2 diabetes without peripheral neuropathy. The tibial nerve (dotted lines indicate) is oval in shape with a honeycomb-like structure visible inside, posterior tibial artery $(\triangle)$, posterior tibial vein $(\mathbf{A})$, tibialis posterior tendon $(\star)$ and flexor digitorum longus $(\boldsymbol{\xi})$.

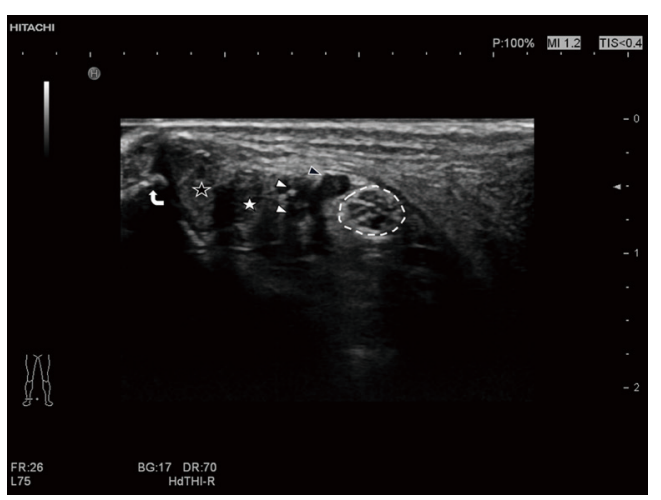

Figure 5 A 57-year-old man with type 2 DPN and the tibial nerve edema. Ultrasound shows the nerve (dotted lines indicate) is thickened and round with a honeycomb-like structure inside., posterior tibial artery $(\triangle)$, posterior tibial vein $(\boldsymbol{\Delta})$, tibialis posterior tendon $(\star)$ and flexor digitorum longus ( $)$. DPN, diabetic peripheral neuropathy.

test (NET), MNSI, and the Toronto clinical scoring system (TCSS), the diagnostic criteria have not yet been unified (7-14). At present, the most objective, sensitive and reliable method to diagnose DNP is NET. NET electrophysiological examination is the gold standard for diagnosis, but the operation is complicated and costly $(15-19)$, and if it is used as a routine examination item, patient compliance is poor. The screen efficiency of MNSI for DPN is better than that of the TCSS and the MNSI has the same diagnostic consistency with NET. The MNSI scoring system is a relatively quick and simple scoring tool for DPN. So it can be used for clinical and epidemiological screen and evaluation of DPN. Compared with other evaluation methods, MNSI has the advantages of strong reliability, simple and quick operation, and good feasibility, making it more suitable for early large-scale screening of DPN (6,20-26). In this study, MNSI was used for screening and grouping.

Previously most experts and researchers believed that the above four ultrasonographic morphological parameters (width; thickness; cross-sectional area; honeycomb structure) were statistically significant in the diagnosis of DPN (27-29). However, the results of this study showed that the thickness and cross-sectional area of the tibial nerve in high-frequency ultrasound characteristics were risk factors, while the width and unclear honeycomb-like structure of the tibial nerve was not.

Ultrasound examination in the DPN group showed that the thickness and cross-sectional area of the tibial nerve increase to varying degrees, which was further confirmed in this study. The sensitivity of tibial nerve thickness is close to that of its cross-sectional area, while the specificity of the cross-sectional area is higher than that of thickness. In the clinical setting, nerve measurement needs to be repeated many times, while thickness measurement is relatively simple. In this study, the OR value of the tibial nerve thickness in this model was 5.176, and the OR value of the cross-sectional area was 1.659 , and DPN was 5.176 times more common in subjects with tibial nerve thickness changes than in subjects without tibial nerve thickness changes. DPN was 1.659 times more common in subjects with tibial nerve cross-sectional area changes than in those without cross-sectional area changes. In this model, the thickness of the tibial nerve was used as the criterion to draw the ROC curve. The Youden index was 0.818 , and $3.6 \mathrm{~mm}$ was selected as the cut-off value to obtain higher sensitivity and specificity. In recent years, with the development of a large number of basic research, our understanding of the pathogenesis of peripheral neuropathy is constantly improving. The study showed that the metabolic mechanism, immune mechanism, genetic mechanism and the influence mechanism of hypoglycemic drugs were all related to the peripheral neuropathy. Nerve biopsy shows axonal damage and in severe cases varying degrees of demyelination and remyelination, axonal injury, and inflammatory cell infiltration. In randomized controlled trials, the risk factors of DPN were increased BMI, triglyceride and hypertension (30,31).

DPN is characterized by the early absence of obvious 
symptoms, a long course of disease, and many confounding factors. It is often difficult to perform RCT studies on the ultrasonographic morphological features of DPN $(32,33)$, and previous studies have shown different results using different inclusion methods and observation target values. The following are the results of studies by different authors: (I) Ishibashi et al. divided diabetic patients into by into five stages: a control group $(n=40)$, stage $I(n=47)$, stage II $(n=72)$, stage III $(n=48)$, and stage IV $+V(n=33)$, to compare differences in the cross-sectional area of the tibial nerve between each group, and the results showed that there was no statistical correlation between HbA1C level and peripheral nerve cross-sectional area (34). The cross-sectional area of the tibial nerve is closely related to the severity of diabetes, and neurological changes can be detected using ultrasound even before the onset of neuropathy. (II) Singh et al. divided subjects into three groups based on the TCSS, which saw a control group $(\mathrm{n}=75)$, NDPN group $(\mathrm{n}=58)$, and DPN group $(\mathrm{n}=75)$. The results of the study suggested that the cross-sectional area and maximum thickness of the tibial nerve in patients with DPN were larger than in healthy non-diabetic controls (35). (III) Singh et al. enrolled 37 patients with DPN and found the mean cross-sectional area of the posterior tibial nerve $3 \mathrm{~cm}$ above the medial malleolus was higher in patients with moderate to severe DPN as compared to that in patients with mild DPN ( $\mathrm{V} \leq 42 \mathrm{~m} / \mathrm{s}$ or $\mathrm{L} \geq 4.4 \mathrm{~ms}$ on nerve conduction study) (36). However, the small sample size in that study may have contributed to the results not holding statistical significance.

Compared with previous studies, this model gives the following different results. The width of the tibial nerve and the unclear honeycomb-like structure were not risk factors in this model, which may be because of neuroanatomical or pathophysiological factors. The nerve bundle structure includes three layers of inner membrane, perineum, and outer membrane, which have protective and regenerating effects. The measurement of tibial nerve width has a large dispersion, and its increase is small, and sensitivity is low when edema occurs. The diagnostic sensitivity of tibial nerve width is only $52.27 \%$, and the specificity is $61.36 \%$. The tibial nerve and its branches below the ankle joint also innervate the skin and plantar muscles, and plantar paresthesia is an early manifestation of DNP (37-39). MNSI-PE evaluates nerve cell function and nerve conduction damage through ankle reflexes, toe vibration, and light touch sensation. An unclear honeycomb-like structure is the main manifestation of the neuropathic morphological changes of the tibial nerve, and changes in its function appear earlier than changes in morphology $(36,40)$. For the evaluation of tibial nerve cell function and nerve conduction impairment, MNSI-PE is more sensitive. Photoshop software has been previously used to conduct quantitative analysis and research on neural brightness. However, choosing different neural brightness cut-off values will result in different research results, and the research methods need to be unified (41).

Differential diagnosis and clinical treatment principle of Diabetic polyneuropathy. Peripheral neuropathy is a general term for a class of diseases caused by structural and functional damage of peripheral nerves. Clinical features of peripheral neuropathy are sensory dysfunction, motor dysfunction, autonomic dysfunction, and reduced or lost tendon reflexes. Peripheral neuralgia variants were divided into primary peripheral neuropathy and secondary peripheral neuropathy, $80 \%$ of which were secondary peripheral neuropathy. Secondary peripheral neuropathy is the main manifestation of sensory nerve dysfunction, and the common diseases are metabolic disorders, infections, amyloidosis, autoimmune diseases, paraneoplastic diseases and genetic diseases. The premise of secondary peripheral neuropathy is primary disease, and there is a close pathological relationship between them. Tracing the primary disease is the method of distinguishing DPN. Type 2 diabetes is the most common cause of secondary peripheral neuropathy. Around the world, type 2 diabetes leads to peripheral neuropathy which is the most common condition. A preventative treatment plan for peripheral neuropathy is to glycemic control and foot care until the onset of pain. However, there is insufficient evidence that prevention strategies are effective in reducing foot symptoms in patients. Pain management, while proven to be effective in clinical randomized controlled trials, does not reverse the outcome of peripheral neuropathy (42).

The novel idea of the paper: (I) the researchers established 1:1 conditional logistics regression model. In this study, general clinical indicators (age, BMI, calf circumference, and gender) were obtained by $1: 1$ approximation matching. This method can eliminate the influence of confounding factors to the greatest extent and improve the reliability of the test results. The selected data will be screened to establish a logistics regression model to find the risk factors of DPN. (II) The opposite risk of risk factors (width, thickness, cross-sectional area, honeycomb structure) and their diagnostic value were compared. (III) MNSI ( $>2$ points) was used in this study as the standard to 
judge type 2 DPN. ROC curve was drawn to find the best diagnostic cut-off value of tibial nerve thickness for DPN.

One limitation of this study is that the tibial nerve is very small, and its measurement requires considerable experience, ability, and compliance with operating specifications. Other limitations concern the retrospective and single center nature of the study, and future research should involve multi-center randomized controls

It is relatively convenient to measure the thickness of the tibial nerve in the clinical setting. On the premise of standardized tibial nerve ultrasound examination and evaluation, its thickness and cross-sectional area are risk factors for diagnosing DPN, and when the thickness is close to $3.6 \mathrm{~mm}$, the sensitivity and specificity will be higher.

\section{Acknowledgments}

Funding: None.

\section{Footnote}

Reporting Checklist: The authors have completed the STARD reporting checklist. Available at https://dx.doi. org/10.21037/apm-21-1573

Data Sharing Statement: Available at https://dx.doi. org/10.21037/apm-21-1573

Conflicts of Interest: All authors have completed the ICMJE uniform disclosure form (available at https://dx.doi. org/10.21037/apm-21-1573). The authors have no conflicts of interest to declare.

Ethical Statement: The authors are accountable for all aspects of the work in ensuring that questions related to the accuracy or integrity of any part of the work are appropriately investigated and resolved. The study was conducted in accordance with the Declaration of Helsinki (as revised in 2013). This study was reviewed and approved by the Ethics Committee of North China Medical Treatment Health Group Fengfeng General Hospital (20201-4-11), and all subjects participated in the study with informed consent.

Open Access Statement: This is an Open Access article distributed in accordance with the Creative Commons Attribution-NonCommercial-NoDerivs 4.0 International License (CC BY-NC-ND 4.0), which permits the non- commercial replication and distribution of the article with the strict proviso that no changes or edits are made and the original work is properly cited (including links to both the formal publication through the relevant DOI and the license). See: https://creativecommons.org/licenses/by-nc-nd/4.0/.

\section{References}

1. Boules ML. Endocavitary versus linear array highfrequency probe in ultrasound-guided supraclavicular subclavian vein central access. Anesth Essays Res 2018;12:873-8.

2. Khalaf KM, Khudhair MS, Ashor AW. Vitamin B12 status and peripheral neuropathy in patients with type 2 diabetes mellitus. J Pak Med Assoc 2019;69:S40-4.

3. Selvarajah D, Kar D, Khunti K, et al. Diabetic peripheral neuropathy: advances in diagnosis and strategies for screening and early intervention. Lancet Diabetes Endocrinol 2019;7:938-48.

4. Cole JB, Florez JC. Genetics of diabetes mellitus and diabetes complications. Nat Rev Nephrol 2020;16:377-90.

5. Gibbons $\mathrm{CH}$. Diabetes and metabolic disorders and the peripheral nervous system. Continuum (Minneap Minn) 2020;26:1161-83.

6. Kaymaz S, Alkan H, Karasu U, et al. Turkish version of the Michigan Neuropathy Screening Instrument in the assessment of diabetic peripheral neuropathy: a validity and reliability study. Diabetol Int 2020;11:283-92.

7. Srinivasan S, Pritchard N, Sampson GP, et al. Diagnostic capability of retinal thickness measures in diabetic peripheral neuropathy. J Optom 2017;10:215-25.

8. Zhang GY, Chen YF, Dai WX, et al. Diabetic peripheral neuropathy increases electrical stimulation threshold of sciatic nerve: a prospective parallel cohort study. Diabetes Metab Syndr Obes 2020;13:4447-55.

9. Rao H, Gaur N, Tipre D. Assessment of diabetic neuropathy with emission tomography and magnetic resonance spectroscopy. Nucl Med Commun 2017;38:275-84.

10. Fenaux P, Platzbecker U, Mufti GJ, et al. Luspatercept in patients with lower-risk myelodysplastic syndromes. N Engl J Med 2020;382:140-51.

11. Di Saverio S, Podda M, De Simone B, et al. Diagnosis and treatment of acute appendicitis: 2020 update of the WSES Jerusalem guidelines. World J Emerg Surg 2020;15:27.

12. Ekman L, Thrainsdottir S, Englund E, et al. Evaluation of small nerve fiber dysfunction in type 2 diabetes. Acta Neurol Scand 2020;141:38-46. 
13. Orlando G, Sacchetti M, D'Errico V, et al. Muscle fatigability in patients with type 2 diabetes: relation with long-term complications. Diabetes Metab Res Rev 2020;36:e3231.

14. Huang L, Zhang Y, Wang Y, et al. Diabetic peripheral neuropathy is associated with higher systolic blood pressure in adults with type 2 diabetes with and without hypertension in the Chinese Han population. Can J Diabetes 2020;44:615-23.

15. Amara F, Hafez S, Orabi A, et al. Review of diabetic polyneuropathy: pathogenesis, diagnosis and management according to the consensus of egyptian experts. Curr Diabetes Rev 2019;15:340-5.

16. Baeriswyl M, Taffé P, Kirkham KR, et al. Comparison of peripheral nerve blockade characteristics between nondiabetic patients and patients suffering from diabetic neuropathy: a prospective cohort study. Anaesthesia 2018;73:1110-7.

17. Garcia-Perez E, Schönberger T, Sumalla M, et al. Behavioural, morphological and electrophysiological assessment of the effects of type 2 diabetes mellitus on large and small nerve fibres in Zucker diabetic fatty, Zucker lean and Wistar rats. Eur J Pain 2018;22:1457-72.

18. Lubberding AF, Pereira L, Xue J, et al. Aberrant sinus node firing during $\beta$-adrenergic stimulation leads to cardiac arrhythmias in diabetic mice. Acta Physiol (Oxf) 2020;229:e13444.

19. Tu LL, Sun Q, Wei LL, et al. Upregulation of GABA receptor promotes long-term potentiation and depotentiation in the hippocampal CA1 region of mice with type 2 diabetes mellitus. Exp Ther Med 2019;18:2429-36.

20. Brocco E, Ninkovic S, Marin M, et al. Diabetic foot management: multidisciplinary approach for advanced lesion rescue. J Cardiovasc Surg (Torino) 2018;59:670-84.

21. Calandruccio JH, Thompson NB. Carpal tunnel syndrome: making evidence-based treatment decisions. Orthop Clin North Am 2018;49:223-9.

22. Lázaro-Martínez JL, Tardáguila-García A, García-Klepzig JL. Diagnostic and therapeutic update on diabetic foot osteomyelitis. Endocrinol Diabetes Nutr 2017;64:100-8.

23. Lung CW, Wu FL, Liao F, et al. Emerging technologies for the prevention and management of diabetic foot ulcers. J Tissue Viability 2020;29:61-8.

24. Aktar Reyhanioğlu D, Adiyaman SC, Bektaş M, et al. Validity and reliability of the Turkish version of the Michigan Neuropathy Screening Instrument. Turk J Med Sci 2020;50:789-97.
25. Reynolds EL, Callaghan BC, Banerjee M, et al. The metabolic drivers of neuropathy in India. J Diabetes Complications 2020;34:107653.

26. Singh A, Chauhan A, Goyal P, et al. Neuropathy in prediabetics: is oxidative stress to contribute? J Assoc Physicians India 2019;67:60-4.

27. Collins MP, Dyck PJB, Hadden RDM. Update on classification, epidemiology, clinical phenotype and imaging of the nonsystemic vasculitic neuropathies. Curr Opin Neurol 2019;32:684-95.

28. Lutchmansingh K, Venkatesh YS, Boppana LKT, et al. The slipping slipper sign: a poor man's test for severe diabetic peripheral neuropathy. J Neuromuscul Dis 2020;7:175-81.

29. Marmolejo VS, Arnold JF, Ponticello M, et al. Charcot foot: clinical clues, diagnostic strategies, and treatment principles. Am Fam Physician 2018;97:594-9.

30. Selvarajah D, Wilkinson ID, Fang F, et al. Structural and functional abnormalities of the primary somatosensory cortex in diabetic peripheral neuropathy: a multimodal MRI study. Diabetes 2019;68:796-806.

31. Wei M, Ye X. Feasibility of point shear wave elastography for evaluating diabetic peripheral neuropathy. J Ultrasound Med 2020;39:1135-41.

32. Dimitrova A, Murchison C, Oken B. Acupuncture for the treatment of peripheral neuropathy: a systematic review and meta-analysis. J Altern Complement Med 2017;23:164-79.

33. Meyer-Hamme G, Friedemann T, Greten HJ, et al. ACUDIN - ACUpuncture and laser acupuncture for treatment of DIabetic peripheral Neuropathy: a randomized, placebo-controlled, partially double-blinded trial. BMC Neurol 2018;18:40.

34. Ishibashi F, Taniguchi M, Kojima R, et al. Elasticity of the tibial nerve assessed by sonoelastography was reduced before the development of neuropathy and further deterioration associated with the severity of neuropathy in patients with type 2 diabetes. J Diabetes Investig 2016;7:404-12.

35. Singh K, Gupta K, Kaur S. High resolution ultrasonography of the tibial nerve in diabetic peripheral neuropathy. J Ultrason 2017;17:246-52.

36. Singh Y, Dixit R, Singh S, et al. High resolution ultrasonography of peripheral nerves in diabetic peripheral neuropathy. Neurol India 2019;67:S71-6.

37. Attah FA, Asaleye CM, Omisore AD, et al. Relationship between sonographically measured median nerve crosssectional area and presence of peripheral neuropathy in 
diabetic subjects. World J Diabetes 2019;10:47-56.

38. He Y, Xiang X, Zhu BH, et al. Shear wave elastography evaluation of the median and tibial nerve in diabetic peripheral neuropathy. Quant Imaging Med Surg 2019;9:273-82.

39. Huang H, Wu S. Application of high-resolution ultrasound on diagnosing diabetic peripheral neuropathy. Diabetes Metab Syndr Obes 2021;14:139-52.

40. Jiang W, Huang S, Teng H, et al. Diagnostic performance of two-dimensional shear wave elastography for evaluating

Cite this article as: Wang K, Yu D, Yao T, Zhang S, Wen L, Gu C. Retrospective study of the ultrasound characteristics of the tibial nerve in patients with type 2 diabetic peripheral neuropathy. Ann Palliat Med 2021;10(8):8787-8796. doi: 10.21037/ apm-21-1573 tibial nerve stiffness in patients with diabetic peripheral neuropathy. Eur Radiol 2019;29:2167-74.

41. Singh KP, Gupta K, Kataria N, et al. High-resolution ultrasonography of the sural nerve in diabetic peripheral neuropathy. J Ultrason 2020;20:e83-9.

42. Barrell K, Smith AG. Peripheral neuropathy. Med Clin North Am 2019;103:383-97.

(English Language Editor: B. Draper) 